\title{
40 GHZ ADIABATIC SOLITON GENERATION FROM A DUAL FREQUENCY BEAT SIGNAL USING DISPERSION DECREASING FIBER BASED RAMAN AMPLIFICATION
}

\author{
Ju Han Lee ${ }^{1}$, Taichi Kogure ${ }^{2}$, Young-Geun Han ${ }^{1}$, Sang Hyuck Kim, Sang Bae Lee ${ }^{1}$, \\ and David. J. Richardson ${ }^{3}$ \\ ${ }^{I}$ Photonics Research Center, Korea Institute of Science and Technology (KIST) \\ 39-1 Hawolgok-Dong, Seongbook-Gu, Seoul, Zip:136-791, Republic of Korea \\ TEL: +82-2-958-5719, FAX:+82-2-958-5709,Email: i.h.lee@ieee.org \\ ${ }^{2}$ Information Technology R\&D Center, Mitsubishi Electric Corporation \\ Kamakura 247-8501, Japan \\ ${ }^{3}$ Optoelectronics Research Centre, University of Southampton \\ Highfield, Southampton, SO17 1BJ, United Kingdom
}

Abstract: We demonstrate the adiabatic soliton compression of a dual frequency beat signal using distributed Raman amplification (DRA) in a dispersion decreasing fiber (DDF). A $40 \mathrm{GHz}$ beat signal generated from a $\mathrm{LiNbO}_{3}$ modulator at a driving RF frequency of $20 \mathrm{GHz}$ is compressed into $\sim 2.2 \mathrm{ps}$ soliton pulses using DRA in a $20 \mathrm{~km}$ DDF. The generation of high quality of soliton pulses from the $40 \mathrm{GHz}$ sinusoidal beat signal is readily achieved with a significantly enhanced compression efficiency using DDF based DRA, compared to the case of using a DDF without DRA or a DSF with DRA. 


\section{INTRODUCTION}

All-optical high speed time division multiplexing (OTDM) systems operating at data rates in excess of $40 \mathrm{Gbit} / \mathrm{s}$ requires the development of stable sources of high repetition rate ultrashort optical pulses operating in the $1550 \mathrm{~nm}$ telecommunication band [1]. Among many other methods one very simple and cost-effective approach to high-repetition rate short pulse generation, is to use adiabatic soliton pulse compression of a dual frequency beat signal. In this approach the dual-frequency beat signal is usually obtained by either two separate distributed feedback (DFB) lasers [2] or a combination of a single DFB laser and a $\mathrm{LiNbO}_{3}$ Mach-Zehnder modulator [3]. The Mach-Zehnder modulator based dual frequency generation method is likely to be a more reliable and simple approach since the generated two optical carriers are phase-synchronized and the corresponding beat signal has a very low phase noise [4]. In order to achieve high quality output pulses using adiabatic soliton compression of the frequency beat signal it is essential to ensure that the compression process is as adiabatic as possible. In a simple scheme based on dispersion decreasing fiber (DDF) the dispersion lengths associated with such sinusoidal beat signal dictate the use of multi-tens of km length scale DDF's in order to ensure that sufficiently adiabatic compression is achieved throughout the system. For such device lengths background fiber loss can have a significant impact on the compression process and limit the degree of compression that can be achieved for physically reasonable ranges of dispersion variation. In a distributed Raman amplification (DRA) based scheme multi-tens of $\mathrm{km}$ of fiber is also typically required to obtain the net on-off gain needed for practical values of pump power. Recently several research groups suggested a novel concept of the use of DRA in a DDF to achieve high quality adiabatic soliton compression [5,6]. This approach enhances the compression factor for which high quality adiabatic compression is achieved for seed pulse sources carved by an electro-absorption modulator (EAM), and reduces the pump power requirements relative to those required for pure DRA compression.

In this paper we experimentally demonstrate the use of distributed Raman amplification in a dispersion decreasing fiber for the efficiency enhancement of adiabatic soliton compression of a dual frequency beat signal. We compress a 40 $\mathrm{GHz}$ beat signal generated from a $\mathrm{LiNbO}_{3}$ modulator at a driving RF frequency of $20 \mathrm{GHz}$ into $\sim 2.2 \mathrm{ps}$ soliton pulses using DRA in a $20 \mathrm{~km}$ DDF. The output pulses are then compared to those generated from the DDF without DRA in terms of pulse pedestal level. The same experiments are also performed with a $20 \mathrm{~km}$ DSF for the overall performance comparison. The generation of high quality of soliton pulses from the $40 \mathrm{GHz}$ sinusoidal beat signal is readily achieved with an enhanced efficiency using distributed Raman amplification in a DDF. 
2. EXPERIMENTAL SETUP AND RESULTS

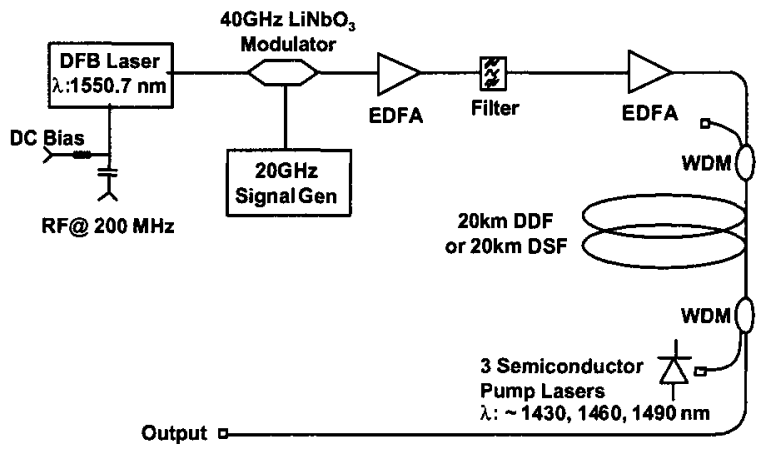

(a)

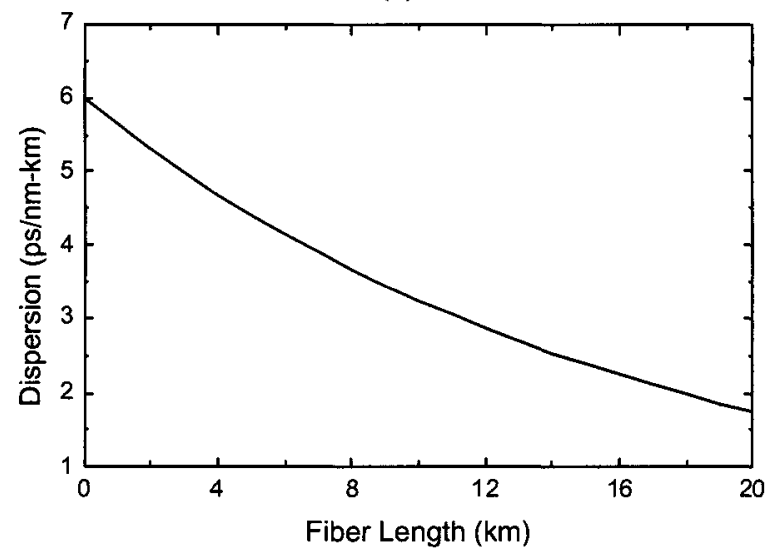

(b)

Figure 1. (a) Experimental setup. (b) Dispersion profile of the dispersion decreasing fiber (DDF) used in this experiment

The schematic of our experiment is shown in Fig. 1(a). A continuous wave (CW) DFB laser was first phase-modulated at a RF frequency of $200 \mathrm{MHz}$ to suppress stimulated Brillouin scattering (SBS) in the dispersion decreasing fiber used in this experiment. The output beam from the DBF laser was then externally modulated using a $\mathrm{LiNbO}_{3}$ Mach-Zehnder modulator which was biased at a null transmission point and driven at a RF frequency of $20 \mathrm{GHz}$ to obtain a $40 \mathrm{GHz}$ sinusoidal optical beat signal. A low-noise erbium doped fiber amplifier (EDFA) followed by a bandpass filter was used to amplify the modulated output beam and another high power EDFA was then employed to boost the optical power of the 
beat signal sufficient to form the first-order soliton at the input end of our DDF. The DDF used in this experiment had a length of $20 \mathrm{~km}$ and its dispersion followed an exponentially tapered profile at $1550 \mathrm{~nm}$ along the length from 6 to $1.75 \mathrm{ps} / \mathrm{nm}$ $\mathrm{km}$ as shown in Fig. 1(b). The dispersion slope was $0.057 \mathrm{ps} / \mathrm{nm}^{2}-\mathrm{km}$. The fiber was originally designed and fabricated for loss-compensated soliton transmission applications and thus the dispersion profile was matched to the power loss of 0.27 $\mathrm{dB} / \mathrm{km}$ within the fiber [7]. Further details concerning the characterization and fabrication of this DDF are provided in Ref. [8]. Three laser diodes operating at center wavelengths of 1430,1460 , and $1490 \mathrm{~nm}$, respectively were employed for the Raman pump. After combing the three pump laser outputs using a fiber based $14 X X / C$-band WDM coupler, a total pump power of up to $500 \mathrm{~mW}$ could be launched into the DDF in a counter-propagating geometry and this level of pump was observed to be able to provide up to $8 \mathrm{~dB}$ of on-off gain within the fiber which was sufficient to compensate for the total background fiber.

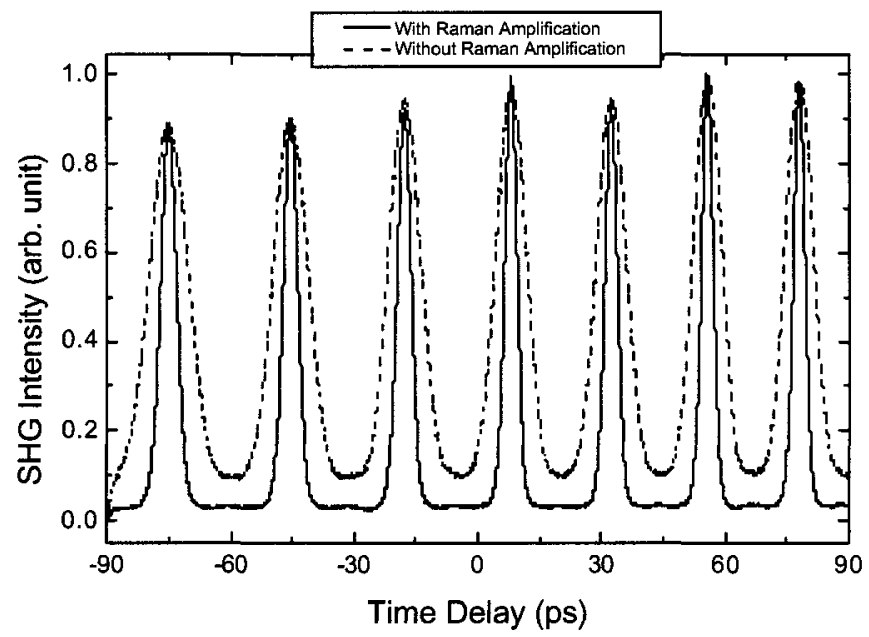

Figure 2. Measured autocorrelation traces of the output compressed pulses after the DDF for both the case with and without distributed Raman amplification.

Fig. 2 shows the measured second harmonic generation (SHG) autocorrelation traces of the compressed pulses after the DDF for both the case with and without DRA. High-quality, low pedestal soliton pulses were obtained with DRA in the DDF whilst both high pedestal level and low compression factor were observed without DRA. Note that the broad background pedestals in the autocorrelation traces are mainly due to the poor extinction ratio of the $\mathrm{LiNbO}_{3}$ modulator used in this experiment and the non-uniform pulse intensity distribution can be attributed to the residual $20 \mathrm{GHz}$ frequency components of the beat signal caused by the 
imperfect DC bias setting of the modulator as shown in the optical spectrum in Fig. 3(b). The temporal shape of the output compressed pulses with DRA in the DDF was observed to be a hyperbolic secant with a full width at half maximum (FWHM) of $2.2 \mathrm{ps}$ as shown in Fig. 3(a). The corresponding optical spectrum of the pulses is shown in Fig. 3(b). The solid line over the measured spectrum shows a least squares sech2 fit, from which the spectral bandwidth was estimated to be about $1.1 \mathrm{~nm}$. The time -bandwidth product of the pulses is 0.31 , indicating that the output compressed pulses are almost transform-limited solitons.

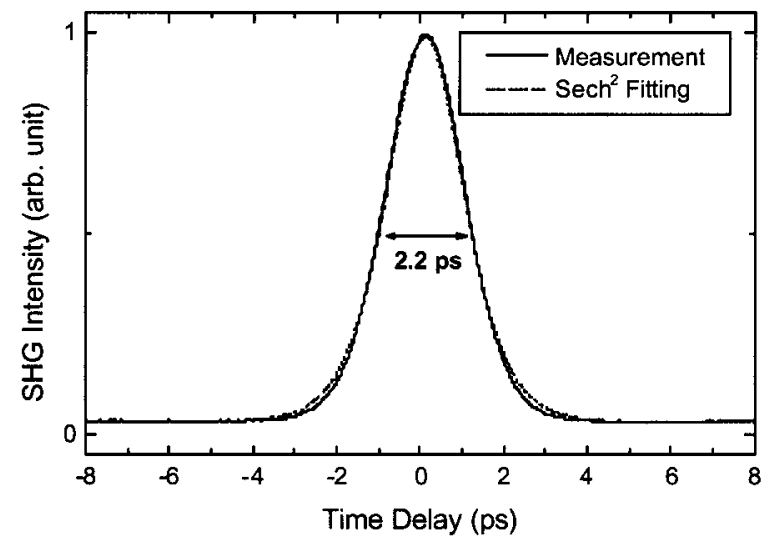

(a)

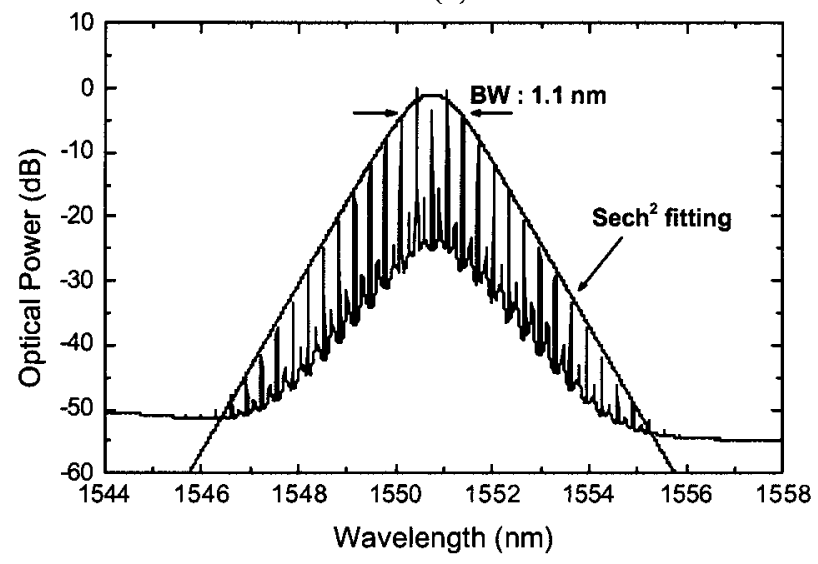

(b)

Figure 3. (a) Measured autocorrelation trace of a single pulse compressed with distributed Raman amplification in the DDF. (b) The corresponding optical spectrum of the puls $\mathrm{e}$. 
In order to confirm the enhanced performance of adiabatic soliton compression using the proposed scheme we performed the same experiment with a $20 \mathrm{~km}$ long dispersion shifted fiber (DSF) which was specially chosen considering the average value of the GVD of the DDF. The GVD of this DSF was $3.8 \mathrm{ps} / \mathrm{nm}-\mathrm{km}$. These results are summarized in Fig. 4. Although distributed Raman amplification along the DSF initiated an adiabatic soliton train formation in some degree, significant pulse compression process was not observed in terms of compression factor and pulse pedestal compared to the case of DRA in the DDF. Without DRA any pulse compression effect was not observed after the DSF. The output pulses after the DSF with DRA was observed to have a Gaussian shape with a FWHM of 5.8 ps. It is clearly evident that sufficient adiabatic soliton compression process was not induced in the DSF despite the use of DRA.

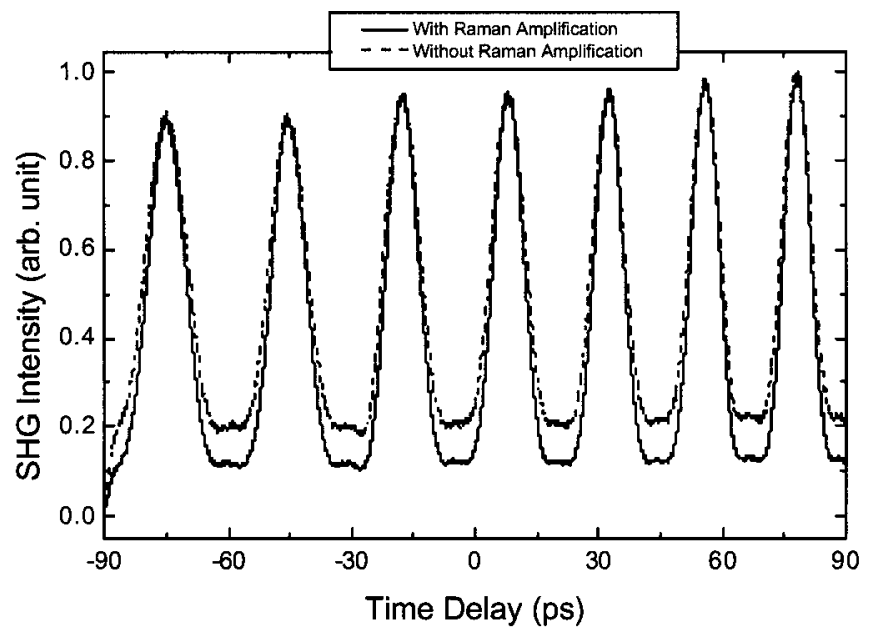

Figure 4. Measured autocorrelation traces of the compressed pulses after a $20 \mathrm{~km}$ long DSF with a GVD of $3.8 \mathrm{ps} / \mathrm{nm}-\mathrm{km}$ for both the case with and without distributed Raman amplification.

\section{CONCLUSION}

We have experimentally demonstrated the efficiency enhancement of adiabatic soliton compression of a dual frequency beat signal using dispersion decreasing fiber based distributed Raman amplification. Highly efficient adiabatic compression of a $40 \mathrm{GHz}$ beat signal generated from a Mach-Zehnder modulator into a $\sim 2.2$ ps soliton pulse train, was achieved using DRA in a $20 \mathrm{~km}$ of DDF. The use of a DDF with an optimized dispersion profile and a high extinction ratio 
$\mathrm{LiNbO}_{3}$ modulator should allow for providing further improvements in terms of compression efficiency and background pedestals .

\section{REFERENCES}

[1] E. Ciaramella, G. Contestabile, A, D'Errico, C. Loiacono, and M. Presi, 'High-power widely tunable $40-\mathrm{GHz}$ pulse source for $160-\mathrm{Gb} / \mathrm{s}$ OTDM systems based on nonlinear fiber effects," IEEE Photon. Technol. Lett., vol. 16, pp.753-755, 2004.

[2] A. V. Shipulin, E. M. Dianov, D. J. Richardson, and D. N. Payne, " $40 \mathrm{GHz}$ soliton train generation through multisoliton pulse propagation in a dispersion varying optical fiber circuit," IEEE Photon. Technol. Lett., vol. 6, pp.1380 - 1382, 1994.

[3] A. D. Ellis, W. A. Pender, T. Widdowson, D. J. Richardson, R. P. Chamberlin, and L. Dong, "All-optical modulation of $40-\mathrm{GHz}$ beat frequency conversion soliton source," Electron. Lett., vol. 31, pp.1362-1364, 1995.

[4] E. A. Swanson, and S. R. Chinn, " $40-\mathrm{GHz}$ pulse train generation using soliton compression of a Mach-Zehnder modulator output," IEEE Photon. Technol. Lett., vol. 7, pp.114-116, 1995.

[5] I. Morita, N. Edagawa, M. Suzuki, S. Yamamoto and S. Akiba, "Adiabatic soliton pulse compression by dispersion decreasing fiber with Raman amplification," in Proc. Opto-Electronics \& Communications Conference, 17P-16, 1996.

[6] T. Kogure, J. H. Lee, and D. J. Richardson, "Wavelength and duration tunable $10 \mathrm{GHz}$, 1.3 ps pulse source using dispersion decreasing fiber based distributed Raman amplification," IEEE Photon. Technol. Lett., vol. 16, pp.1167-1169, 2004.

[7] D. J. Richardson, R. P. Chamberlin, L. Dong and D. N. Payne, "High quality soliton loss-compensation in $38 \mathrm{~km}$ dispersion-decreasing fibre," Electron. Lett., vol. 31, pp.1681-1682, 1995.

[8] N. G. R. Broderick, D. J. Richardson, and L. Dong, "Distributed dispersion measurements and control within continuously varying dispersion tapered fibers," IEEE Photon. Technol. Lett., vol.9, pp.1511-1513, 1997. 